\title{
Two Dimensional Signal Spreading in UMTS LTE: Exploiting Time-Frequency Diversity to Increase Throughput
}

\author{
J. Blumenstein - M. Šimko • R. Marsalek • Z. Fedra • \\ J. Prokopec · M. Rupp
}

Published online: 5 October 2012

(C) The Author(s) 2012. This article is published with open access at Springerlink.com

\begin{abstract}
This paper proposes a two-dimensional spreading method in UMTS Long Term Evolution (LTE). Such a method brings additional time-frequency diversity which is beneficial in terms of the ability to decode transmitted data at the receiver site under multipath fading. Additional diversity is provided due to the fact that chips of transmitted signals are spread using a Walsh-Hadamard spreading sequence over several subcarriers as well as over several time-slots simultaneously. A comparison with state-of-the-art LTE downlink transmission is provided. To enable reproducibility and to increase credibility of our results, an open source Vienna LTE simulator is utilized. LTE transmissions with the proposed 2D spreading are tested on several channel models. Simulations show that apart from an Additive White Gaussian Noise channel model, this 2D spreading based method outperforms the throughput performance of standard LTE. At high Signal to Noise Ratio (SNR), the throughput increase in most channel models is higher than $10 \%$.
\end{abstract}

Keywords LTE · 2D Spreading · Throughput maximization · VSF-OFCDM · Vienna LTE simulator

\section{Introduction}

It is without any doubt that one of the main concerns of any communication system is to have the highest possible data throughput independent of the channel conditions. To maximize the

J. Blumenstein $(\varangle) \cdot$ R. Marsalek $\cdot$ Z. Fedra $\cdot$ J. Prokopec

Department of Radio Electronics, Brno University of Technology, Purkyňova 118, 61200 Brno, Czech Republic

e-mail: xblume00@phd.feec.vutbr.cz

M. Šimko $\cdot$ M. Rupp

Institute of Telecommunications, Vienna University of Technology,

Gusshausstrasse 25/389, A-1040 Vienna, Austria

e-mail: msimko@nt.tuwien.ac.at

M. Rupp

e-mail: mrupp@nt.tuwien.ac.at 
throughput, we exploited time and frequency diversity. Apart from utilization of expensive techniques such as using extra bandwidth, adding more antennas or simply increasing the transmitting power, our method can be interpreted as a coding technique with code rate one. This method only increases the demand on computational power.

The idea behind this paper is to reveal the efficiency of a $2 \mathrm{D}$ spreading method when it is incorporated in the most relevant wireless standard which is currently available. Therefore we chose an open source LTE simulator [1-3] to find out whether LTE can be enhanced by our proposed $2 \mathrm{D}$ signal spreading and show that it indeed outperforms a standard LTE downlink in terms of throughput.

An example of implementation of 2D signal spreading and thus a time-frequency diversity utilization can be found in Variable Spreading Factor Orthogonal Frequency Code Division Multiplex (VSF-OFCDM) systems [4-7]. The authors claim that the 2D spreading based VSF-OFCDM system exhibits a better performance than Orthogonal Frequency Division Multiplexing (OFDM) wireless transmission methods.

A three-cell frequency reuse OFDM system and one-cell reuse VSF-OFCDM system was compared. Due to the fact that VSF-OFCDM systems could benefit from the utilization of the code domain and the cells have been distinguished by a spreading code the VSF-OFCDM system reached significantly better throughput in a multi cell environment [8]. The reason for this is that the possibility to use the entire system bandwidth in the VSF-OFCDM system was enabled when compared to a third of the bandwidth in the case of pure OFDM.

LTE however uses a one-cell frequency reuse [9] and could benefit only from exploitation of the time-frequency diversity. To keep the comparison of 2D LTE and LTE fair, the bandwidth has to be the same as in the case of LTE.

When presenting our novel method, the need for comparing with a-state-the-art system is very important. Hence, the system model is based on the Vienna LTE simulator $[1,2]$ as a reference model. This simulator is released under a free non-commercial, academic use license. Such a release model enables algorithms to be tested and being compared using a common, known, and verifiable environment. We are certain that this transparency not only improves the quality of the published results but also their credibility.

Concerning results, we present throughput comparisons based on power-delay profile (PDP) channel models of the following types: Pedestrian channel model of type A (PedA), Pedestrian channel model of type B (PedB), Vehicular channel model of type A (VehA), Additive White Gaussian Noise (AWGN), Typical urban channel model (TU), Extended typical urban channel model (ETU), Rural area channel model (RA) and Hilly terrain channel model (HT) [10,11].

The remainder of this paper is organized as follows: In the Sect. 2, the implementation of the 2D signal spreading into the LTE signal processing chain is described. In the Sect. 3 we explain its selection and in the Sect. 4 the comparison of the standard LTE downlink and the 2D spreading based LTE transmission is provided. At the end of the paper, the Sect. 5 rounds up the paper.

\section{System Model}

The 2D spreading, as it is presented in [12], is rather repeating symbols which are multiplied by some spreading sequence. The resulting chips are repeated in time, frequency or code domain. The presented algorithm exploits one dimensional Walsh-Hadamard sequences. Nevertheless, due to interleaving of spread data among multiple carrier frequencies and 
Fig. 1 The LTE signal processing chain. The dark gray blocks represents the additional blocks for 2D spreading and despreading. Other parts remain the same in both versions, 2D spreading based LTE and standard LTE. The white blocks are according to [13-15]

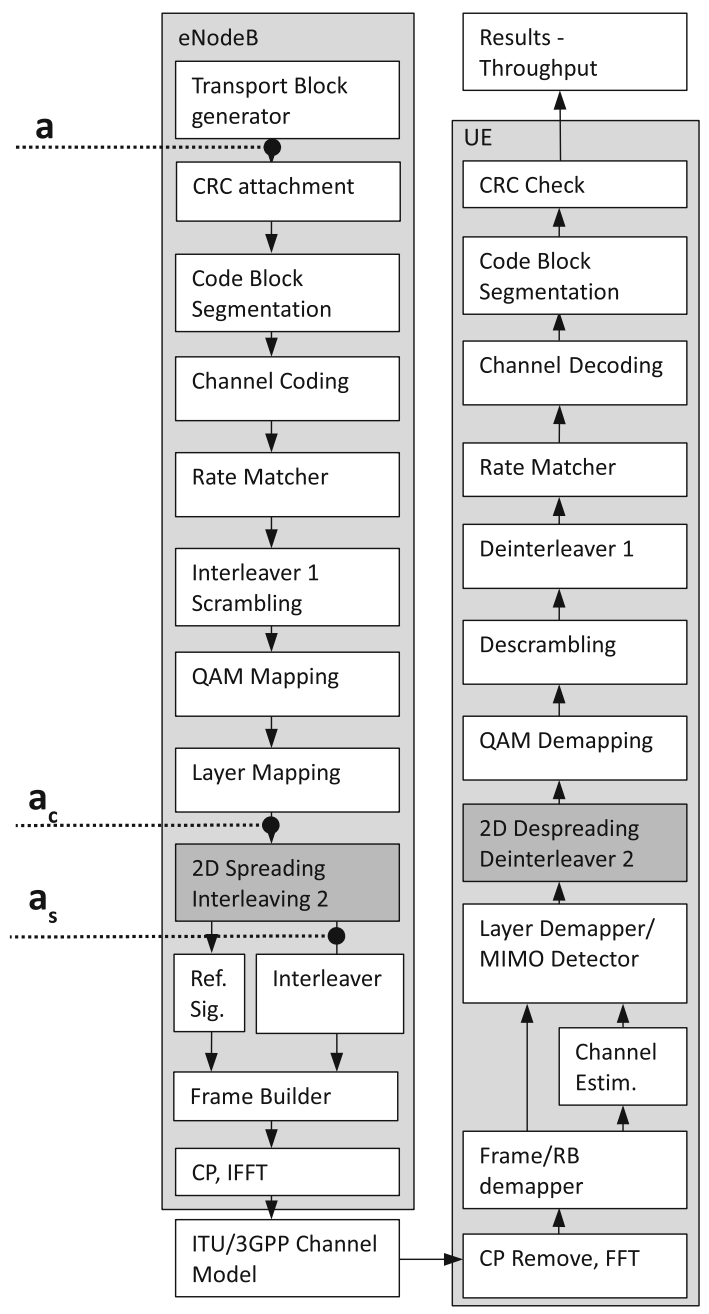

multiple OFDM symbols, two dimensions are utilized. Therefore, we refer to this scheme as two-dimensional spreading.

Figure 1 represents the LTE signal processing chain. The dark gray blocks indicate additional blocks for 2D spreading and despreading. Other parts remain the same in both versions; 2D spreading based LTE and standard LTE downlink. The white blocks are according to 3GPP standards [13-15].

Vector $\mathbf{a}_{\mathbf{c}}$ from Eq. (1) denotes the Cyclic Redundancy Check (CRC) secured, segmented, scrambled, turbo-coded, rate matched, QAM and layer mapped data vector a.

$$
\mathbf{a}_{\mathbf{c}}=\left(a_{\mathrm{c} 1}, a_{\mathrm{c} 2}, \ldots, a_{\mathrm{c} e}\right),
$$

where the index $e$ is the number of elements in the vector $\mathbf{a}_{\mathbf{c}}$. We consider the output of the layer mapping block, vector $\mathbf{a}_{\mathbf{c}}$, as the input of a $2 \mathrm{D}$ spreading block. In the $2 \mathrm{D}$ spreading block, vector $\mathbf{a}_{\mathbf{c}}$ is chopped to SF parts, where SF stands for Spreading Factor-as explained below. This operation is illustrated by (2), where $\left.\mathrm{SP}\left\{\mathrm{a}_{\mathbf{c}}\right\}\right|_{\mathrm{SF}}$ denotes a Serial-to-parallel (SP) transformation of vector $\mathbf{a}_{\mathbf{c}}$. 


$$
\left.\mathrm{SP}\left\{\mathbf{a}_{\mathbf{c}}\right\}\right|_{\mathrm{SF}}:=\underbrace{\left(\begin{array}{cccc}
a_{\mathrm{c} 1} & a_{\mathrm{c} 2} & \cdots & a_{\mathrm{c} \frac{e}{S F}} \\
a_{\mathrm{c}\left(\frac{e}{S F}+1\right)} & a_{\mathrm{c}\left(\frac{e}{S F}+2\right)} & \ddots & \vdots \\
\vdots & \ddots & a_{\mathrm{c}(e-S F-2)} & a_{\mathrm{c}(e-S F-1)} \\
\cdots & a_{\mathrm{c} e}
\end{array}\right)}_{\mathbf{A}_{\mathbf{c}}} .
$$

Each row of matrix $\mathbf{A}_{\mathbf{c}}$ is then multiplied by one Walsh-Hadamard sequence, i.e., one column of matrix $\boldsymbol{\Xi}$ specified by (4). This is described in Eq. (3) and depicted in Fig. 2.

$$
\begin{aligned}
\mathbf{A}_{\mathbf{s}} & =\mathbf{A}_{\mathbf{c}(i, *)} \otimes \boldsymbol{\Xi}_{(*, i)} \\
& =\left(\begin{array}{cccc}
a_{\mathrm{c} 1} \xi_{1,1} & a_{\mathrm{c}\left(\frac{e}{S F}+1\right)} \xi_{1,2} & \ldots & a_{\mathrm{c}(e-S F)} \xi_{1, S F} \\
a_{\mathrm{c} 1} \xi_{2,1} & a_{\mathrm{c}\left(\frac{e}{S F}+1\right)} \xi_{2,2} & \vdots & \vdots \\
\vdots & \vdots & & \\
a_{\mathrm{c} 1} \xi_{S F, 1} & a_{\mathrm{c} 2} \xi_{1,1} & \vdots & \vdots \\
a_{\mathrm{c} 2} \xi_{1,1} & a_{\mathrm{c}\left(\frac{e}{S F}+2\right) \xi_{1,2}} & \\
\vdots & \vdots & a_{\mathrm{c}(e-S F-2)} \xi_{S F-1, S F-1} & \vdots \\
a_{\mathrm{c} \frac{e}{S F} \xi_{S F, 1}} & \vdots & a_{\mathrm{c}(e-S F-1)} \xi_{S F, S F-1} & a_{\mathrm{c} e} \xi_{S F, S F}
\end{array}\right)^{T}
\end{aligned}
$$

where

$$
\boldsymbol{\Xi}=\left(\begin{array}{ccc}
\xi_{1,1} & \ldots & \xi_{1, S F} \\
\vdots & & \vdots \\
\xi_{S F, 1} & \ldots & \xi_{S F, S F}
\end{array}\right) .
$$

Matrix $\boldsymbol{\Xi}$ then represents the Walsh-Hadamard matrix utilized as a bank of spreading sequences, $\mathbf{A}_{\mathbf{c}(i, *)}$ denotes the $i$-th row of matrix $\mathbf{A}_{\mathbf{c}}$ and $\boldsymbol{\Xi}_{(*, i)}$ selects the $i$-th column of matrix $\boldsymbol{\Xi}$. The symbol $\otimes$ denotes the Kronecker product and (. $)^{T}$ denotes a matrix transposition.

Consequently, all the rows of the resulting matrix $\mathbf{A}_{\mathbf{s}}$ are summed together according to Eq. (5), thus we obtain a vector of spread data $\mathbf{a}_{\mathbf{s}}$ of length SF. Each row of matrix $\mathbf{A}_{\mathbf{c}}$ forms the code sheet, as shown in Fig. 2.

$$
\begin{aligned}
& \mathbf{a}_{\mathbf{s}}=\sum_{j=1}^{S F} \mathbf{A}_{\mathbf{s}(j, *)} \\
& =\left[\begin{array}{ccc}
a_{\mathrm{c} 1} \xi_{1,1}+a_{\mathrm{c} 1} \xi_{2,1}+ & \cdots & +a_{\mathrm{c} \frac{e}{S F} \xi_{S F, 1}} \\
a_{\mathrm{c}\left(\frac{e}{S F}+1\right) \xi_{1,2}+a_{\mathrm{c}\left(\frac{e}{S F}+1\right)} \xi_{2,2}+\cdots} & \cdots & \\
\vdots & \cdots & +a_{\mathrm{c}\left(\frac{e}{S F}+1\right)} \xi_{S F, 2}+a_{\mathrm{c}\left(\frac{e}{S F}+2\right)} \xi_{1,2} \\
a_{\mathrm{c}(e-S F)} \xi_{1, S F}+ & \cdots & +a_{\mathrm{c}(e-S F-1)} \xi_{S F, S F-1}
\end{array}\right]^{T}
\end{aligned}
$$

In order to spread the chips (the elements of vector $\mathbf{a}_{\mathbf{s}}$ ) over the entire bandwidth and over all time slots (OFDM symbols), all positions of the chips are randomly interleaved. 


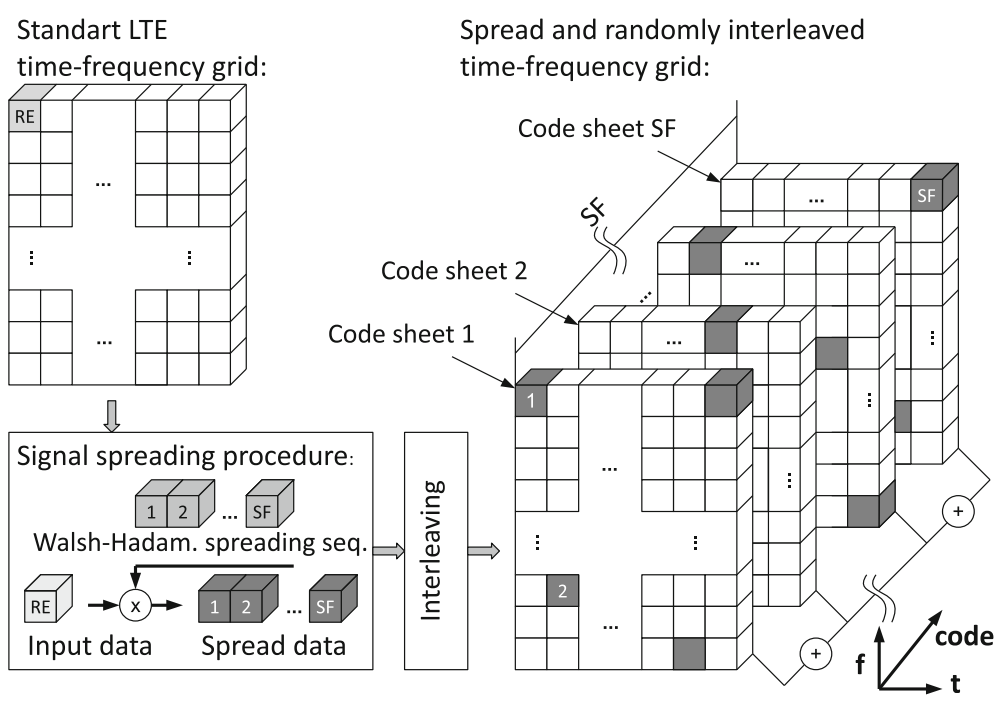

Fig. 2 The two-dimensional signal spreading process. We are spreading the signal right after the layer mapping block. The signal from the layer mapper is multiplied with all Walsh-Hadamard sequences of order SF. As a result we receive SF vectors, which represents the code-sheets. These are then summed together and we obtain a vector which serves as a frame builder input

Vector $\mathbf{a}_{\mathbf{s}}$, containing all the spread data is of the same length as the output of the layer mapping block, i.e., $\left\|\mathbf{a}_{\mathbf{s}}\right\|=\left\|\mathbf{a}_{\mathbf{c}}\right\|$.

Due to the fact that Walsh-Hadamard sequences are orthogonal, we are able to separate them at the receiver site. This principle is well-known from the Code Division Multiple Access (CDMA) systems [12].

When spreading a signal, we have SF-times more of data to transmit due to the spreading process, however, we also obtain SF times more space in the code domain which we can utilize. The increase in terms of data is in this case the same as the increase of the space dedicated to the transmission. As a result, after the summation of all the code sheets as presented in Fig. 2, the amount of data is kept the same as in the case of standard LTE and thus no extra bandwidth is required.

However, the transmitted data occupies SF-times more time-frequency space, thus we gain on time-frequency diversity. At the receiver site, we have SF realizations of one bit. Due to the interleaving of these chips all over the transmission channel, the influence of deep fades of a transfer function [16] will be reduced.

The data share their space with other SF - 1 data element. It will be shown in the next section that their performance on AWGN channels will not be improved.

\section{Spreading Factor}

For a better understanding of our proposed algorithm, a brief description of the LTE timefrequency signal grid according to [14] is stated in the following section. Using a 'normal' cyclic prefix, seven symbols with 12 subcarriers each form one resource block. One element of such a grid is called resource element (RE). 
The maximal number of resource blocks transmitted in LTE is given by a total system bandwidth BW, where BW $\in\{1.4,3,5,10,15,20\} \mathrm{MHz}$. This corresponds to the number of resource blocks $\mathrm{N}_{r b} \in\{6,15,25,50,75,100\}$, which can be exploited for data transmission.

The spreading factor SF has to be chosen carefully, the length of the vector $\mathbf{a}_{\mathbf{c}}$ representing the amount of transmitted data has to be divisible by SF. This is due to the fact that the 2D spreading block cuts its input vector $\mathbf{a}_{\mathbf{c}}$ into SF parts in order to allow spreading. For a more detailed explanation, the chopped data are multiplied by the spreading sequence and then their length will be SF-times larger. The length of the spread data will then be exactly the same as the length of the $2 \mathrm{D}$ spreading block input vector $\mathbf{a}_{\mathbf{c}}$. This is a necessary property. The frame builder can remain intact as well as the generation of pilot signals used for channel estimation. As a result, only minimal changes in the LTE standard are needed. Additionally, the comparison of such a system is more relevant.

In the configuration presented below, where $\mathrm{BW}=1.4 \mathrm{MHz}$, the number of user equipments nUE $=1, \mathrm{~N}_{r b}=6$, a vector from the layer mapping block is of length 816 for the first subframe and 960 for the second subframe, respectively. Due to this, the maximal spreading factor is $\mathrm{SF}=48$. The reason is that 48 is the highest common divider of 816 and 960 for which we also found a Walsh-Hadamard matrix.

\section{Experiment and Results}

This section describes the simulation setup as well as the results of the 2D spreading based LTE model.

Our method operates on the physical layer. Therefore, for a lucidity of our experiment, we assume a perfect time synchronization of all User Equipments (UEs) which is provided by a higher layer. The resulting throughput curve, seen in Fig. 4, is cell-specific and the scheduling of resource blocks is done by a scheduler which is also part of the higher layer of LTE functionality. If we consider that the whole available bandwidth is scheduled to one UE, we can simplify the transmission scheme as the single user environment as seen in Fig. 3.

Regardless the Number of User Equipments (nUE), but of course only when nUE $>1$, our simulation setting generates maximal cell-specific data throughput given by the system bandwidth.

Figure 4 depicts the comparison of a standard compliant LTE downlink transmission with a non standard compliant 2D LTE downlink. Adaptive Channel Quality Information (CQI) mapping's, for which the CQI is adapted in order to provide the highest possible data throughput at given SNR, are utilized. The setup of the simulation presented in Fig. 4 is as follows: PedB channel model, 1.4 MHz, single-user, 5,000 subframes, CQIs from 1 to $15, \mathrm{SF}=48$.

Fig. 3 For a lucidity of our experiment, we assume a perfect timing synchronization. If we consider that the whole available bandwidth is scheduled for a single user, here UE1, and omitting additional users (UE2 and UE3), we have simplified the transmission scheme. Thus we obtain a cell-specific throughput curve as seen in Fig. 4

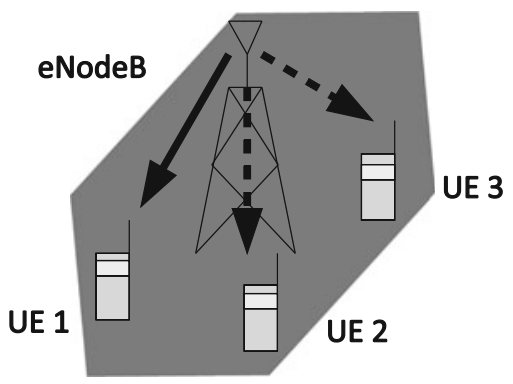


Fig. 4 The throughput curves for PedB channel model, 1.4 MHz, single-user, 5,000 sub-frames, CQIs from 1 to 15 , $\mathrm{SF}=48$. The $2 \mathrm{D}$ spreading based LTE is plotted in red, the standard LTE is dashed and blue. The $99 \%$ confidence intervals are also depicted, indicating a high confidence of the plotted results. (Color figure online)

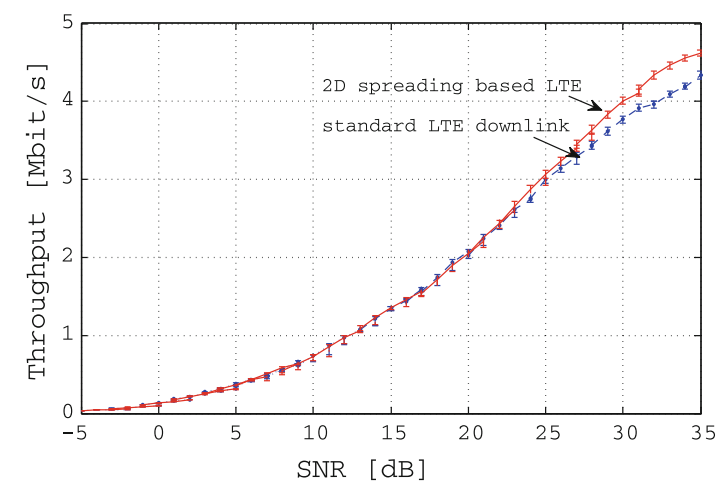

\begin{tabular}{ll}
\hline System bandwidth & $1.4 \mathrm{MHz}$ \\
Subcarrier spacing & $15 \mathrm{kHz}$ \\
Subframe duration & $1 \mathrm{~ms}$ \\
Number of user equipments (UEs) & 1 \\
Number of eNodeBs & 1 \\
Transmission scheme & SISO \\
Number of subframes & 10,000 \\
Channel quality information (CQI) & 15 \\
SNR & $30 \mathrm{~dB}$ \\
SF & 48 \\
CP length & 'Normal' [13] \\
Channel estimation method & MMSE \\
Channel model & PedA, PedB, VehA, AWGN, TU, \\
& ETU, RA, HT [10,11] \\
\hline
\end{tabular}

Table 1 LTE system parameters of the presented simulations 
Fig. 5 The throughput increase for various channel models and for $\mathrm{SNR}=30 \mathrm{~dB}$. Simulation settings are listed in Table 1. The error bars represents $99 \%$ confidence intervals

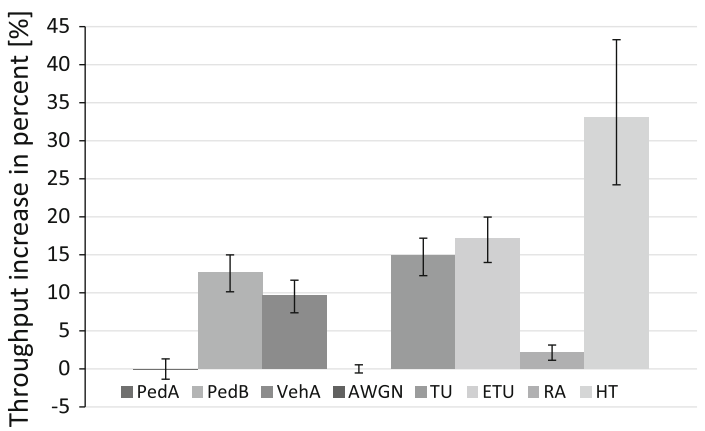

\section{Conclusion}

This paper describes a new 2D spreading mapping suitable for LTE transmissions. We implemented this algorithm into the state-of-the art simulation system developed at Vienna University of Technology. For a comparison of the proposed 2D spreading enhanced LTE and standard LTE downlink, several channel models have been applied.

The proposed spreading requires only minor modifications of LTE standards while bringing considerable improvement in the throughput performance of such systems. We showed that in most channel models, the utilization of the 2D spreading is beneficial in terms of throughput increase. This increase ranges from 5\% to more than $25 \%$ (99\% confidence interval). It should also be noted, that the exploitation of the $2 \mathrm{D}$ spreading does not require extra bandwidth.

Acknowledgments This work has been funded by the Christian Doppler Laboratory for Wireless Technologies for Sustainable Mobility, KATHREINWerke KG, and A1 Telekom Austria AG. The financial support by the Federal Ministry of Economy, Family and Youth and the National Foundation for Research, Technology and Development is gratefully acknowledged. The Grant Agency of Czech Republic grant no. P102/10/P513 "Communication systems combining OFDM and CDMA and their PAPR" is also gratefully acknowledged. The authors would like to thank Josep Colom Ikuno for many fruitful and interesting discussions on this and related topics. The research published in this submission was financially supported by the project CZ.1.07/2.2.00/20.0007 WICOMT of the operational program Education for competitiveness.

Open Access This article is distributed under the terms of the Creative Commons Attribution License which permits any use, distribution, and reproduction in any medium, provided the original author(s) and the source are credited.

\section{References}

1. LTE simulator homepage. [Online]. Available: http://www.nt.tuwien.ac.at/ltesimulator/

2. Mehlführer, C., Wrulich, M., Ikuno, J. C., Bosanska, D., \& Rupp, M. (2009). Simulating the long term evolution physical layer. In European signal processing conference (EUSIPCO). Scotland: Glasgow, August 2009.

3. Mehlführer, C., Colom Ikuno, J., Simko, M., Schwarz, S., Wrulich, M., \& Rupp, M. (2011). The Vienna LTE simulators-Enabling reproducibility in wireless communications research. in EURASIP Journal on Advances in Signal Processing, 2011(1), 29. [Online]. Available: http://asp.eurasipjournals. com/content/2011/1/29.

4. Maeda, N., Kishiyama, Y., Atarashi, H., \& Sawahashi, M. (2003). Variable spreading factor-ofcdm with two dimensional spreading that prioritizes time domain spreading for forward link broadband wireless access. in Vehicular technology conference, 2003. VTC 2003-Spring. The 57th IEEE semiannual (Vol. 1, pp. 127-132), April 2003. 
5. Atarashi, H., Maeda, N., Abeta, S., \& Sawahashi, M. (2002). Broadband packet wireless access based on VSF-OFCDM and MC/DS-CDMA. In The 13th IEEE international symposium on personal, indoor and mobile radio communications, 2002 (Vol. 3, pp. 992-997), September 2002.

6. Maeda, N., Atarashi, H., Abeta, S., \& Sawahashi, M. (2002). Throughput comparison between VSFOFCDM and OFDM considering effect of sectorization in forward link broadband packet wireless access. In Vehicular Technology Conference, 2002. Proceedings. VTC 2002-Fall. 2002 IEEE 56th (Vol. 1, pp. 47-51).

7. Blumenstein, J., Fedra, Z., \& Sebesta, V. (2010). Performance of pilot aided channel estimation technique in 2D spreading based systems. Radioengineering, 19, 507-510.

8. Chong, C.-C., Watanabe, F., Inamura, H., Wang, D., Minn, H., \& Al-Dhahir, N. (2008). On the performance comparison of VSF-OFCDM and OFDM. In IEEE 19th international symposium on personal, indoor and mobile radio communications, 2008 (PIMRC 2008) (pp. 1 -6), September 2008.

9. Sesia, S., Toufik, I., \& Baker, M. (2009). LTE, The UMTS long term evolution: From theory to practice. New York: Wiley.

10. ITU IMT-2000. (1997). Recommendation ITU-R m.1225: Guidelines for evaluation of radio transmission technologies for IMT-2000. Technical report.

11. 3GPP. (2007). Technical specification group radio access network; deployment aspects (release 7). 3GPP, Technical report 25.943 V7.0.0, January 2007.

12. Fazel, K., \& Kaiser, S. (2008). Multi-carrier and spread spectrum systems: From OFDM and MC-CDMA to LTE and WiMAX. Wiley. [Online]. Available: http://books.google.com/books?id=RzUJsPqe-dgC

13. 3GPP Technical Specification Group RAN. (2009). E-UTRA; physical channels and modulation. 3GPP, Technical report TS 36.211 Version 8.7.0, May 2009.

14. 3GPP Technical Specification Group RAN. (2009). E-UTRA; multiplexing and channel coding. 3GPP, Technical report TS 36.212, March 2009.

15. 3GPP Technical Specification Group RAN. (2009). E-UTRA; physical layer procedures. 3GPP, Technical report TS 36.213, March 2009.

16. Hanzo, L. (2003). OFDM and MC-CDMA for broadband multi-user communications, WLANs, and broadcasting. Wiley. [Online]. Available: http://books.google.com/books?id=hQ6bl3RG04sC

\section{Author Biographies}

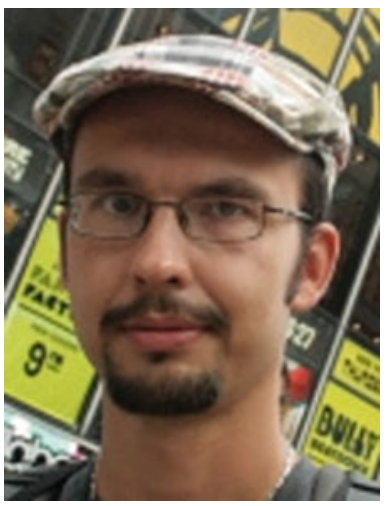

Jiri Blumenstein received his Master degree in electrical engineering at the Brno University of Technology in 2009. In 2011 he worked with Josep Colom Ikuno and Michal Šimko at the Institute of Telecommunications, Vienna University of Technology on LTE simulations. At present, he is a PhD student at the Department of Radio Electronics, Brno University of Technology. His research interests are communication systems based on combination of OFDM and CDMA. 

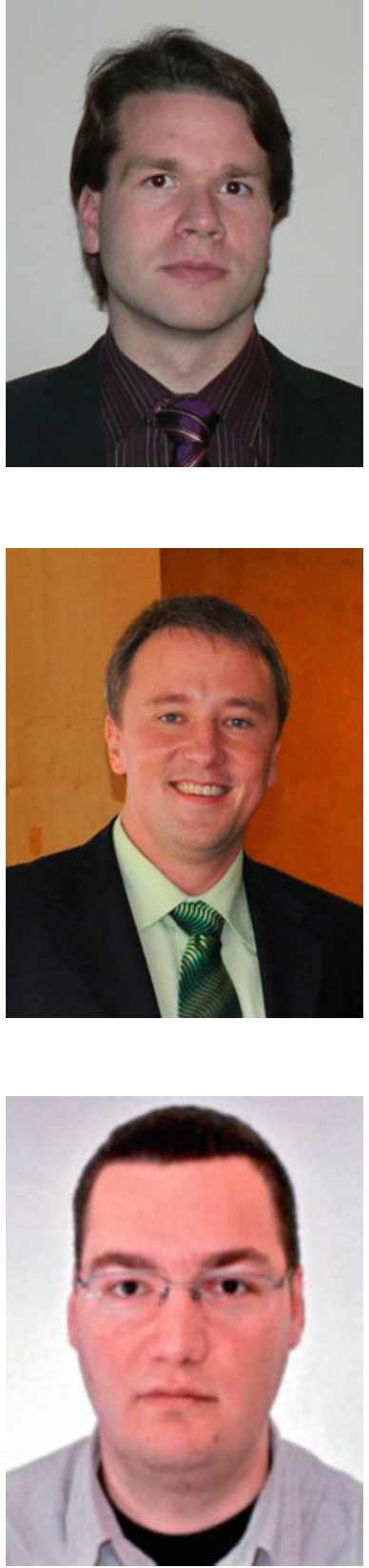

Michal Šimko was born in Bratislava, Slovakia, in October 1985. In June 2009 he received his Dipl.-Ing. degree (with highest honors) in electrical engineering from the Vienna University of Technology. Since August 2009 he is working toward his $\mathrm{PhD}$ as project assistant at the Institute of Telecommunications, Vienna University of Technology. In June 2011, he received bachelor degree in industrial-organizational and social psychology from the Comenius University in Bratislava. Michal has been awarded the Best Student Paper Award by IEEE VTS Society at IEEE VTC 2011 Spring in Budapest.

Roman Maršálek graduated at the Brno University of Technology in 1999 and received the doctoral degree from Université de Marne-LaVallée, École Supérieure d'Ingénieurs en Électronique et Électrotechnique de Paris, France in 2003. He is currently assistant professor at the Department of Radio Electronics, Brno University of Technology in the Czech Republic. His research interests are in wireless communications theory and applied digital signal processing.

Zbynek Fedra was born in Dacice, Czech Republic in 1981. He graduated from Brno University of Technology in 2004 and received the Ph.D. degree in 2008. He is currently assistant professor at Department of Radio Electronics, Brno University of Technology. His research interests are multicarrier communication and signal processing. 

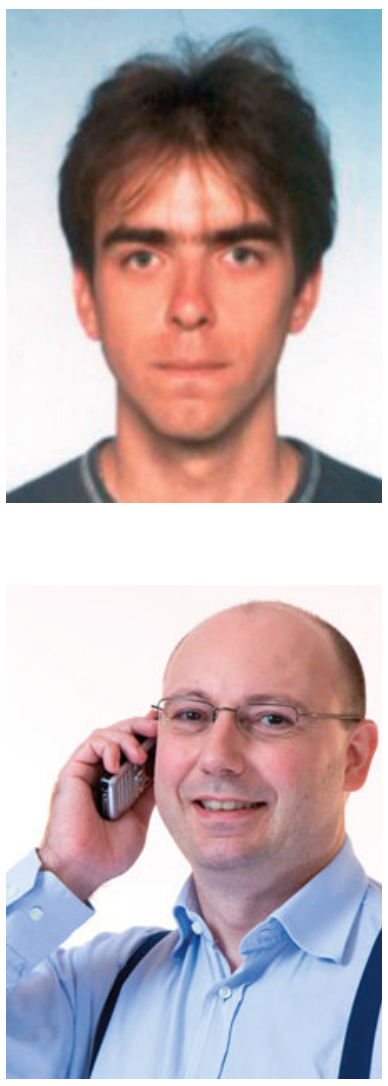

Jan Prokopec was born in Rychnov nad Knìžnou, Czech Republic in 1978. He graduated at Brno University of Technology in 2001, where he also received the Ph.D. in 2006. He is currently assistant professor at Department of Radio Electronics, Brno University of Technology. His research interests are mobile communications systems and parallel programming. He is an IEEE member since 2003.
Markus Rupp received his Dipl.-Ing. degree in 1988 at the University of Saarbrücken, Germany and his Dr.-Ing. degree in 1993 at the Technische Universitaet Darmstadt, Germany, where he worked with Eberhardt Haensler on designing new algorithms for acoustical and electrical echo compensation. From November 1993 until July 1995, he had a postdoctoral position at the University of Santa Barbara, California with Sanjit Mitra where he worked with Ali H.Sayed on a robustness description of adaptive filters with impact on neural networks and active noise control. From October 1995 until August 2001 he was a member of Technical Staff in the Wireless Technology Research Department of Bell-Labs at Crawford Hill, NJ, where he worked on various topics related to adaptive equalization and rapid implementation for IS-136, 802.11 and UMTS, including the first MIMO prototype for UMTS. Since October 2001 he is a full professor for Digital Signal Processing in Mobile Communications at the Vienna University of Technology where he founded the Christian-Doppler Laboratory for Design Methodology of Signal Processing Algorithms in 2002 at the Institute for Communciations and RF Engineering. He served as Dean from 2005 to 2007. He was associate editor of IEEE Transactions on Signal Processing from 2002 to 2005, is currently associate editor of JASP EURASIP Journal of Advances in Signal Processing and JES EURASIP Journal on Embedded Systems. He is elected AdCom member of EURASIP since 2004 and served as president of EURASIP from 2009 to 2010. He authored and co-authored more than 400 scientific papers and patents on adaptive filtering, wireless communications, and rapid prototyping, as well as automatic design methods. 